\title{
Face-based constructive approach in 3D environment to facilitate the interpretation of multiview orthographic projection to future engineers
}

\author{
V. Pérez-Belis, V. Gracia- Ibáñez, R. Plumed, M. Gómez-Fabra, M.J. Agost, A. Piquer, \\ C. González-Lluch, M. Núñez-García
}

* Universitat Jaume I, Castellón de la Plana, España

Departamento de Ingeniería Mecánica y Construcción

\begin{abstract}
One of the main objectives of Engineering Graphics' syllabus is to improve the capacity of spatial vision of students, as well as providing essential teaching of graphic representation techniques. In this course, students should create and interpret engineering drawings. However, a significant number of them have problems interpreting and understanding multiview orthographic projections. The course is commonly studied at first year of several engineering Bachelor's Degrees. In this course, the capacity of spatial vision is critical, as students have to interpret a real object from different projection methods. Nevertheless, this is not an obvious task for most students, since either they have no prior training or traditionally this training has no effect on student's capability of reflexion and interpretation. We consider that facilitating the object interpretation may involve a significant educational improvement. For this reason, in this work we present the approach and development of a specific method to interpret an object. It is focused on harnessing the three dimensional (3D) environment of AutoCAD ${ }^{\circledR}$ software which is used in the course, for the face-based construction of objects.
\end{abstract}

Keywords: multiview visualization; teaching strategy; face-based constructive approach.

\section{Introduction}

As McGrath \& Brown outlined in their White Paper (McGrath \& Brown, 2005), to develop appropriate skills in the fields of science and engineering visual learning is necessary. These skills, facilitate the collaborative learning and develop the students' creativity. We consider that the inclusion of the visual thinking in the curriculum of engineering education is needed.

At first sight, it was easy to think that the advent of available 3D methods would imply the annihilation of the previous two-dimensional methods-well established throughout the twentieth century as 'Projection Methods'. In recent years, 3D was incorporated in academic environments generally in Bachelor's Degrees. This approach has coexisted, in many engineering areas, with the traditional approach (pencil and paper) being complemented with digital work in $2 \mathrm{D}$ previous to incorporate the $3 \mathrm{D}$, by means of orthographic and axonometric projections.

Obtaining the orthographic views from the axonometric projection of an object and vice versa were, and still are, core activities in any basic course of Engineering Graphics. 3D commercial software could easily obtain 2D main projections from a 3D model, but it is unable to interpret an engineering object from its orthographic projection views where the highest difficulty lies.

'The ability to visualize objects in three dimensions is a key skill for engineering design. Physical models for proposed designs often do not exist, and an engineer must be able to envision, manipulate, and modify designs when they are presented in the form of 2-D drawings', this is a transcript from Chapter 11 'Advanced Visualization Techniques', of 'Visualization, modeling and graphics for Engineering Design' (Lieu \& Sorby, 2008), which is characterized by assuming the need to updating the graphic fundamentals for engineering. The authors show different methods to obtain the 
axonometric two-dimensional projections from the orthographic projection with high level of detail.

The aim of this work is focused on how students can reach the comprehension of the orthographic views represented. This is made by means of providing a new method that organizes systematically, step by step, under certain conditions, the construction of a 3D model of an object starting from the orthographic views. This method partially agrees with the section "Strategy for Constructing Pictorials by Inverse Tracking of Surfaces" described in the previously cited work. This is not the only influence in this work. In addition to our daily academic life in contact with engineering students, we would like to point out contributions to this work from nearby universities to the city where the Congress is performed (Melgosa Pedrosa, 2012; Mugika, 2010), as well as more distant sources (Suh \& McCasland, 2009). In all of them, as in many other proposals, it can be found common aspects to our approach. At minimum all agree in the desire to improve the learning experience in the field of Engineering Graphics.

The most common 3D construction system used is the Constructive Solid Geometry (CSG), which is not the one used in this work. Instead, the method uses a construction system by faces, consisting in sealing all the external surfaces of the 3D object, like a B-Rep. The comprehension of the object is by adding material (in an affirmative way). Unlike the negative way (Gómez-Fabra, 2007) which has the purpose of identify the geometry by looking for volumes without material and removing them.

After the appearance of 3D technology, several research groups arose using artificial intelligence resources to face this problem. In case of success, this would imply a necessary reorientation of graphical fundamentals. Although our approach does not correspond with that ambitious line of work (Company, Piquer, Contero, \& Naya, 2005; Dori \& Tombre, 1995), it highlights the intrinsic difficulty of the merits of the problem referred in this communication.

\section{Method}

The method consists on the construction of the object through 3D tools, by going step by step from their orthographic views. It provides the students a global understanding through the concordance of these orthographic views resulting in the final construction of the 3D object. It is considered that this method can be a key factor for students who do not have the innate ability of spatial vision, as shown in Figure 1.
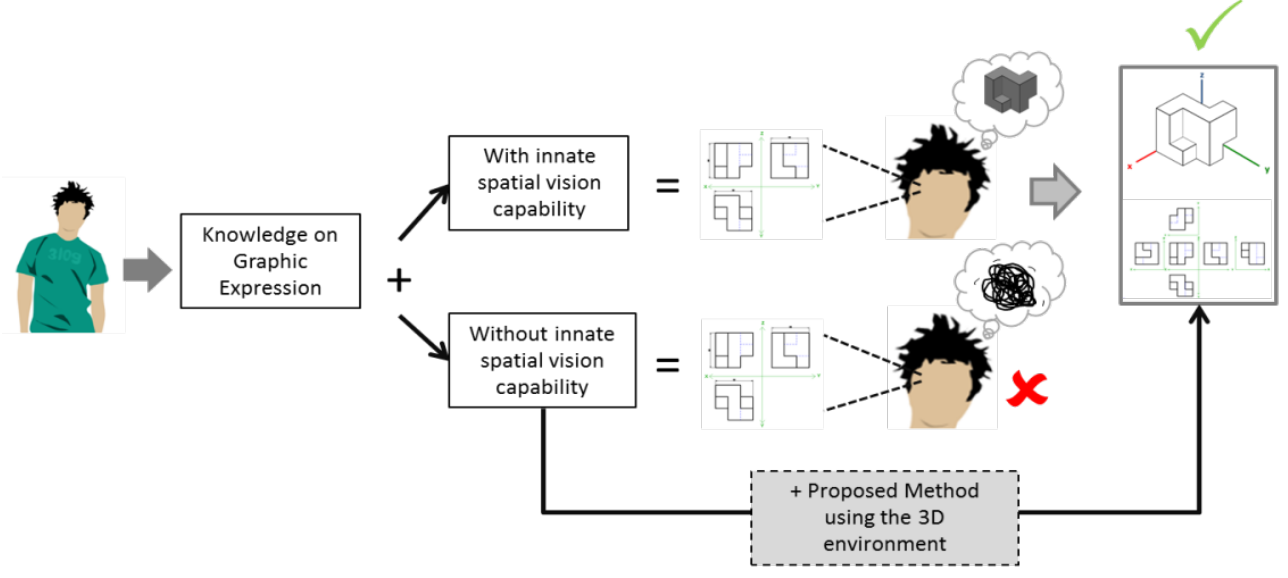

Figure 1. Context of the method implementation. 
The method is implemented with the software AutoCAD® 2015, which is used in teaching Engineering Graphics. It consists on the steps listed below in Table 1.

Table 1. Detailed description of the method.

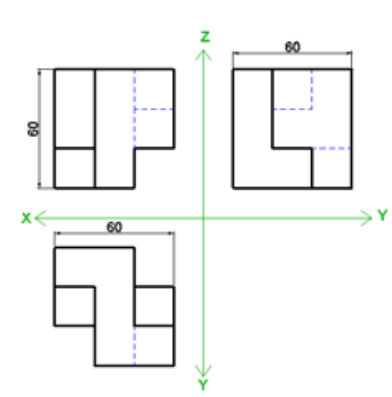

1. The starting point corresponds to the orthographic views in European system, drawn in a $2 \mathrm{D}$ environment. It is essential to cut the drawing lines into segments whenever there is an intersection, in order to be able to select any closed circuit as a face.

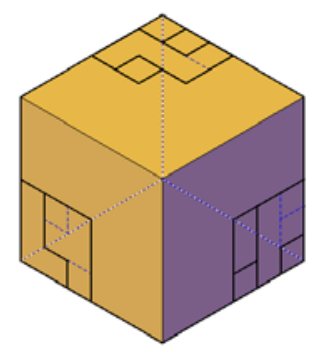

3. Next, the views are copied on the corresponding faces of the prism (in their accurate 3D position). ${ }^{3}$

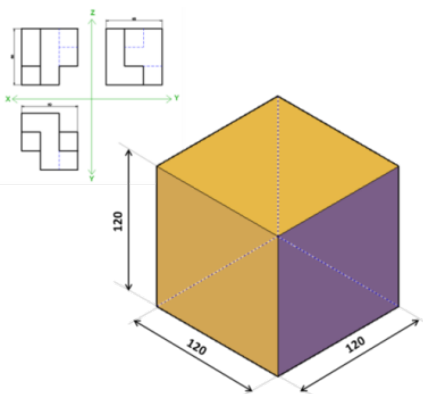

2. In the workspace "3D Modeling”, a prism is created. Its maximum dimensions are twice of the maximum dimensions of the orthographic views according to the three axes. ${ }^{1}$ This allows the students to work in a more comfortable way. ${ }^{2}$

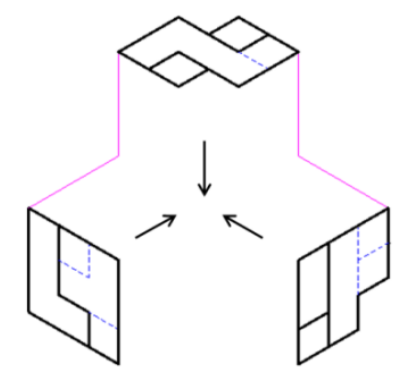

4. Once each face is located, the prism is hidden and the views are analyzed with the objective of creating the object step by step, according to the concordance of the views.

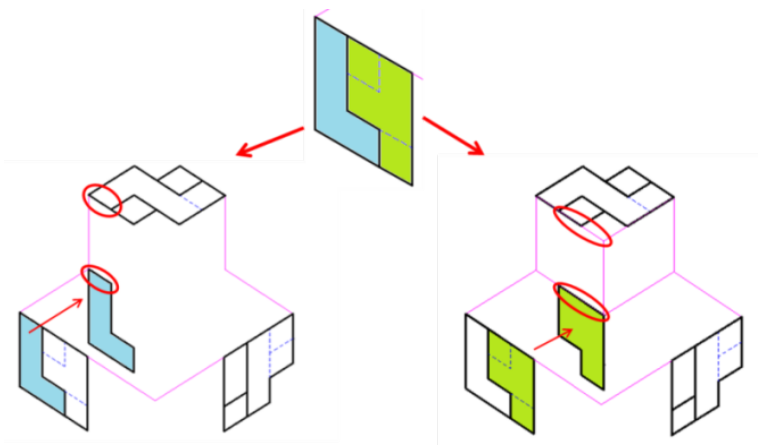

5. First, external faces should be created on the object from the initial orientation. To do this, from each external face, closed circuits without continuous interior lines should be identified. After that, these circuits should be copied and converted into regions. These regions will shape the solid faces of the object to work with. ${ }^{4}$

6. In this case, we start by analyzing the side view. It is shown that there are two circuits without continuous interior lines, so there are two possibilities to place the faces in the first position. However, if the top view it is observed, no concordance between the side and the top view is presented, therefore, this face should not be in the first position.

\footnotetext{
${ }^{1}$ AutoCAD provides the workspace "3D Modeling" where it is possible to define the box. This could be created through the "box" command or with the multiple tools that AutoCAD offers.

${ }^{2}$ In order to visualize the faces of the object, "Conceptual Visual Style" should be activated.

${ }^{3}$ In order to place each view on each face, it is necessary to copy and paste them directly $(\mathrm{Ctrl}+\mathrm{shift}+\mathrm{c} / \mathrm{ctrl}+\mathrm{v})$ without forgetting to maintain activated dynamic UCS.

${ }^{4}$ The regions are created using the "region” command from the group “drawing”. These entities are key in the process.
} 


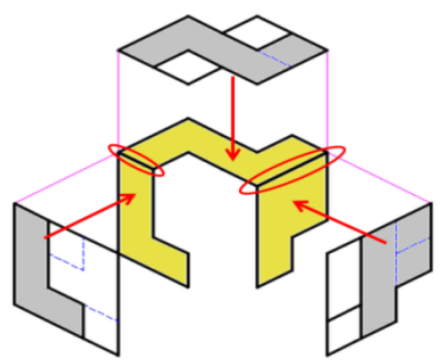

7. Following systematically this process, external faces are created.

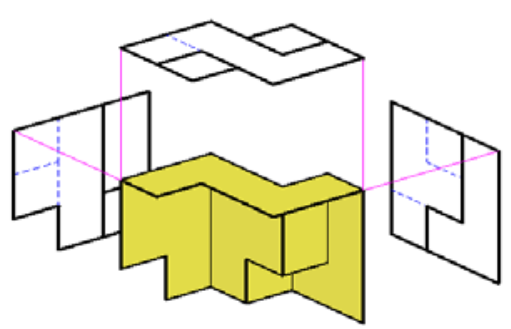

9. Once each side is finished, the object orientation is changed until the remaining sides are created.

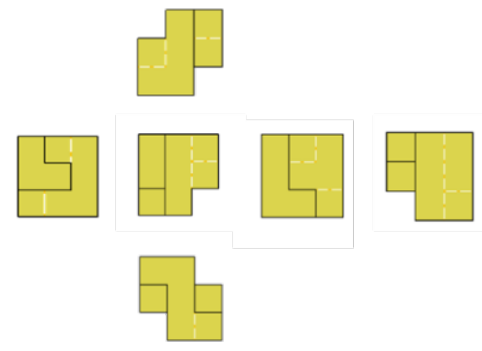

11. After finishing the object we obtain a full 3D model of the original object created face by face. Orientation can be changed to see the different orthographic views which could be drawn and checked with the original ones provided on this problem. $^{5}$

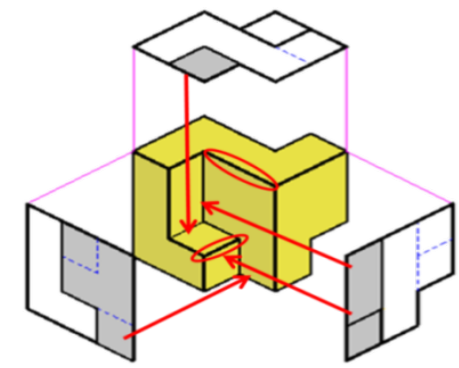

8. Next, the object is finished using a coherence pattern on the views, starting from external faces edges.
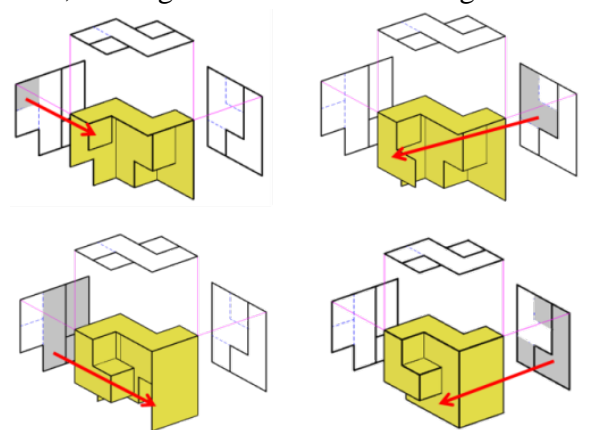

10. In this particular case, it is essential to analyze all hidden edges as they provide valuable information about the object, including hidden faces so it will be necessary to analyze its concordance.

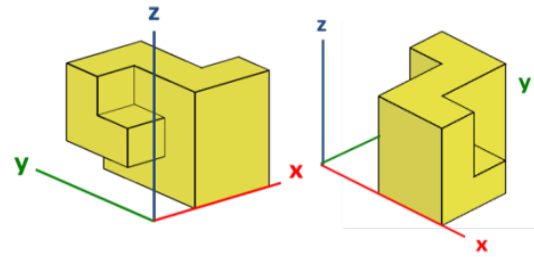

12. In addition, the intended axonometric projection can be obtained using 3D modeling tools and changing its orientation. Taking those views as an starting point any axonometric projection needed could be drawn. ${ }^{5}$

The purpose of this method is to provide a tool to interpret objects through the construction of 3D model, without losing perception details by the student when he is creating the object from the given views. This is why the objects are created side by side instead of the traditional solid pattern CSG.

This method is primarily conceived to construct polyhedral objects since the guidelines of the course are focused on planar surfaces before introducing curves. It could also be used to construct any polyhedral object whose faces are parallels to the projection plane. If those faces are not parallel to the projection planes, the steps to follow are shown in Table 2.

\footnotetext{
${ }^{5}$ From object's interpretation, any orthographic view and axonometric projections can be drawn. Also they can be extracted as a 2D representation in a block by using "Flatshot" located in the group "Section". AutoCAD does not allow too much flexibility in the "Layout" workspace, so obtaining those representations are not immediate.
} 
Table 2. Example of polyhedral object whose faces are not parallel to the projection planes.

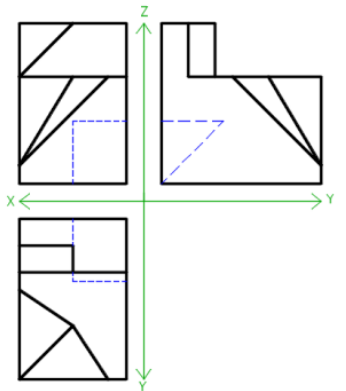

1. The method starts from the orthographic views of the object.

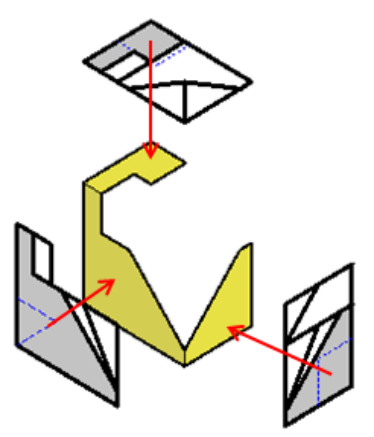

3. The process starts with the external faces.

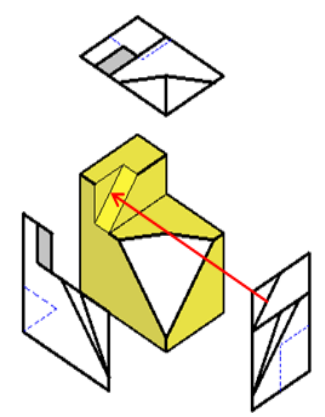

5. If faces are not parallel to coordinate planes but they are included in a projection plane on any of them, parallel edges have to be copied. ${ }^{6}$

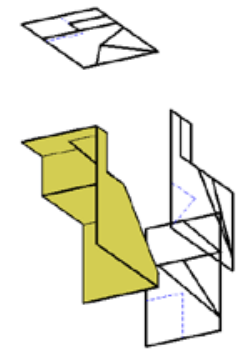

7. In order to visualize the backside of the object, orientation system should be changed.

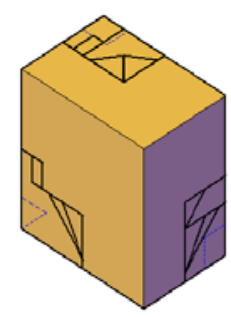

2. According to the previous explanation, the orthographic views have to be located on the corresponding faces of the box.

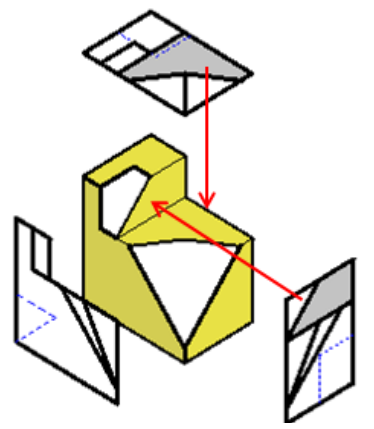

4. Successively, it has to be applied to secondary faces

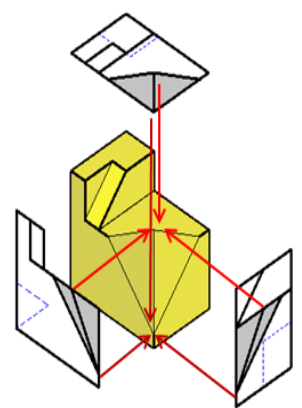

6. If faces are not parallel to any coordinate plane, edges should be outlined from their starting and ending point. ${ }^{6}$

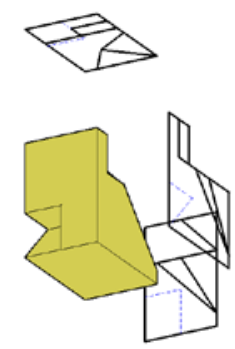

8. The process continues until the object is completely closed.

\footnotetext{
6 To construct the object "face-by-face", the current user coordinate system (UCS) should be the same as the orientation of the face, in order to create the region. This region is the result of a polyline which has been converted into a region.
} 


\section{Results and Discussion}

A method to facilitate the interpretation of multiview through the construction of the object "face by face" is proposed since a lack in methods to help students without inborn visualization skills it is aimed to be covered. AutoCAD ${ }^{\circledR}$ is the software used because it is the one available for students to be checked. Currently, this method it is being put to the test. Unfortunately, thus far quantitative data about its effectiveness are not available, however, some results from a simple tests show a quite good acceptance among students.

\section{Conclusions}

Spatial vision capability is one of the skills needed in subjects related to Engineering Graphics, but if students do not have this ability within their innate capacities, it would be desirable to be developed or improved through different methods and tools. Since the main problem of students seems to be the orthographic views interpretation, this paper presents a method to scaffold them to understand different objects from their orthographic multiviews through some 3D tools.

The method, helps the students to understand, step by step, the different stages of the process improving the comprehension of the object, since they are continuously linking the two dimensional projections with the three dimensional representation. This process results especially interesting in this subject since the objects of the problems tend to be irregular and far from the reality even helping in that case those students with visualization abilities.

Future lines of research could include the consideration of the construction of objects with curved surfaces.

\section{References}

Company, P., Piquer, A., Contero, M., \& Naya, F. (2005). A survey on geometrical reconstruction as a core technology to sketch-based modeling. In Computers and Graphics (Pergamon) (Vol. 29, pp. 892-904). doi:10.1016/j.cag.2005.09.007

Dori, D., \& Tombre, K. (1995). From engineering drawings to 3D CAD models: are we ready now?

Gómez-Fabra, M. (2007). Colaboración de un programa 3D en la lectura diédrica y obtención del modelo. In Congresso Internazionale Congiunto XVI ADM - XIX INGEGRAF Congreso Internacional Conjunto XVI ADM - XIX INGEGRAF Perugia, 6 - 8 Giugno 2007 (pp. 6-8).

Lieu, D., \& Sorby, S. (2008). Visualization, Modeling, and Graphics for Engineering Design (p. 1088). Delmar Celgage Learning. Retrieved from http://books.google.com/books?hl=ca\&lr=\&id=lHO79rg6VT8C\&pgis=1

McGrath, M.B., \& Brown J.R. (2005). Visual learning for science and engineering. Computer Graphics and Applications IEEE.vol. 25(5), pp. 56-63.

Melgosa Pedrosa, C. (2012). Diseño y eficacia de un gestor web interactivo de aprendizaje en ingeniería gráfica para el desarrollo de la capacidad de visión espacial. Retrieved from http://riubu.ubu.es//handle/10259/178

Mugika, E. S. U. and M. G. (2010). New Achievements in Technology Education and Development. Chapter 8. Methodology for Part Visualization Problem Solving - the Importance of the Process. In S. Soomro (Ed.), . InTech. doi:10.5772/219

Suh, Y. S., \& McCasland, J. (2009). Interactive construction of solids from orthographic multiviews for an educational software tool. Computer-Aided Design and Applications, 6, 219-229. doi:10.3722/cadaps.2009.219-229 